Classification

Physics Abstracts

$66.10-72.20-73.90-77.55$

\title{
Injection d'ions dans le cyclohexane entre électrodes recouvertes de couches minces isolantes
}

\author{
N. Nemamcha, B. Gosse, J. P. Gosse et A. Denat \\ C.N.R.S. Laboratoire d'Electrostatique et de Matériaux Diélectriques (*), 166 X, 38042 Grenoble Cedex, \\ France
}

(Reçu le 22 décembre 1986, révisé le 9 avril 1987, accepté le 13 avril 1987)

\begin{abstract}
Résumé. - La conduction de solutions d'électrolytes dans le cyclohexane a été étudiée entre électrodes recouvertes de films d'alumine ou de polyphénylène-oxyde lors de l'application d'un échelon de tension continue. L'injection faible observée aux temps courts dans les solutions de TlAPi a les mêmes caractéristiques qu'entre électrodes métalliques nues (même variation avec le champ, avec la conductivité de la solution). Les ions injectés sont les ions de l'électrolyte, ils sont créés dans la zone de force-image. Aux temps longs le film qui bloque les ions du liquide, devient un injecteur très fort d'ions dans le liquide. La nature de ces ions n'a pas été déterminée.
\end{abstract}

\begin{abstract}
The electrical conduction of electrolyte solutions in cyclohexane has been studied between metal electrodes coated with thin films of alumina or polyphenyleneoxide. The ionic injection observed at short times after the application of a voltage step presents the same characteristics as the one previously studied between metallic electrodes (same field dependence, same variation with the liquid conductivity). We conclude that the injected ions are the ions of the electrolyte and are created in the region of image-force in the liquid. After a long application of the voltage, the coating which has blocked the ions of the electrolyte, behaves as a strong injector of ions in the liquid.
\end{abstract}

\section{Introduction.}

Le courant de conduction et les pertes électriques des liquides diélectriques augmentent très rapidement quand le champ électrique dépasse environ $100 \mathrm{kV} \mathrm{cm}^{-1}$. Nous avons montré qu'il y a alors injection d'ions par les électrodes et que ce phénomène est amplifié quand la conductivité du liquide est élevée [1].

Pour déterminer le mécanisme d'injection, nous avons étudié des solutions de l'électrolyte TIAPi (picrate de triisoamylammonium) dans le cyclohexane $(\mathrm{CH})$ en fonction de différents paramètres tels que le champ électrique, la température, la conductivité de la solution. L'injection répond au schéma réactionnel général :

$$
\mathrm{C}+\mathrm{e}^{-\underset{k_{\mathrm{r}}}{\stackrel{k_{\mathrm{f}}}{\leftrightarrows}}} \mathrm{C}_{\mathrm{i}}^{-\stackrel{k_{\mathrm{ex}}}{\rightarrow}} \mathrm{C}_{\mathrm{l}}^{-}
$$

Ce schéma a été introduit la première fois pour expliquer l'injection de trous dans des cristaux

(*) Laboratoire Associé à l'Univ. Scient. Techn. et Méd. de Grenoble. organiques isolants à partir de solutions aqueuses [2]. Il comprend une étape (1) de création d'ions $\mathrm{C}^{-}$à l'électrode qui correspond au transfert électronique entre le métal et le liquide. Les ions ainsi créés (indice i) sont soumis à la force-image et n'entrent dans le volume du liquide (indice l) qu'après avoir franchi la barrière de potentiel de force-image.

Dans les solutions de TlAPi dans le cyclohexane, nous avons constaté expérimentalement que la densité de courant injecté est de la forme $j=A k_{\mathrm{ex}}(E)$ où $k_{\mathrm{ex}}$ est la constante de vitesse d'extraction des ions hors de la zone de force-image. Ceci signifie que l'étape d'extraction est l'étape lente du phénomène, elle lui impose sa cinétique fonction du champ électrique.

Le coefficient de proportionnalité qui est relié à l'étape de création d'ions par les constantes cinétiques $k_{\mathrm{f}}$ et $k_{\mathrm{r}}$ est trouvé indépendant de $E$, mais proportionnel à la densité $q_{0}$ en ions libres dans le liquide. Cette particularité est expliquée par le fait que les ions injectés sont les ions de l'électrolyte [4]. Dans le travail précédent [3], nous avons étudié l'influence de la nature de l'additif sur la conduction des hydrocarbures. Selon l'électrolyte et le rayon 
hydrodynamique de ses ions, on observe ou non une injection. Mais, quand l'injection prédomine, on constate toujours que $j \propto k_{\text {ex }}(E) q_{0}$.

Dans le présent travail, nous avons voulu modifier plus profondément les caractéristiques de la réaction (1) de transfert d'électron en recouvrant les électrodes d'une couche mince isolante. Cette couche, soit minérale $\left(\mathrm{Al}_{2} \mathrm{O}_{3}\right)$ soit organique (polyphénylène-oxyde), a été élaborée par technique électrochimique. Nous présentons dans le chapitre 2 la technique de préparation de ces couches, leurs propriétés physiques et électriques dans des structures métal/isolant/métal. Nous décrivons ensuite l'influence de ces couches sur la conduction de solutions de TIAPi dans le cyclohexane. Compte tenu des caractéristiques des couches et du liquide, les phénomènes suivant les premiers instants de l'application de la tension (quelques temps de transit des ions dans le liquide) sont caractéristiques de l'état de l'interface couche isolante/liquide avant la mise sous tension. Ce sera l'objet du chapitre 3 . Dans le chapitre 4, nous décrivons la conduction lors d'une aplication prolongée de la tension, ce sont alors les constantes de vitesse des réactions de transfert d'électrons métal/liquide et d'extraction hors de la zone de force-image (schéma (1)) qui devraient déterminer la densité de courant stationnaire. Enfin dans le chapitre 5, nous discuterons nos résultats.

\section{Théorie.}

La densité de courant d'injection dans un liquide non polaire contenant un électrolyte, par exemple du cyclohexane contenant du TIAPi, entre électrodes planes, parallèles, métalliques s'écrit [4] :

$$
j=K q_{0} \exp \left(\varnothing_{\mathrm{e}} / U\right) \exp \left(-x_{\mathrm{A}} E / U\right) G(E) .
$$

$E$ est le champ électrique supposé uniforme entre les électrodes, $U=k T / e, x_{\mathrm{A}}$ est la distance minimale d'approche des ions du métal, $\varnothing_{\mathrm{e}}$ est la différence de potentiel dans la zone de charge d'espace proche du métal (couche diffuse), $K$ est la mobilité des ions injectés, $q_{0}$ est la densité volumique d'ions libres dans la solution en l'absence de champ électrique.

La fonction $G(E)$ s'écrit

$$
G(E)=E / 2 X_{\mathrm{B}} K_{1}\left(2 X_{\mathrm{B}}\right)
$$

où $K_{1}$ est la fonction de Hankel de la variable $X_{\mathrm{B}}=(e E / 4 \pi \varepsilon)^{1 / 2} / 2 U$.

L'expression (2) de la densité de courant est obtenue en supposant que la distribution de charges à l'interface n'est pas modifiée par le champ appliqué. Si l'on écrit le schéma réactionnel général (1) sous la forme :

$$
\mathrm{TIAPi}+\mathrm{e}^{-\underset{k_{\mathrm{r}}}{\stackrel{k_{\mathrm{f}}}{\leftrightarrows}}} \mathrm{TIA}+\left[\mathrm{Pi}^{-}\right]_{\mathrm{i}}^{k_{\mathrm{ex}}}\left[\mathrm{Pi}^{-}\right]_{1}
$$

en accord avec les résultats expérimentaux, ceci signifie qu'entre les constantes de vitesse existe la relation $k_{\text {ex }} \ll k_{\mathrm{r}}$ [TIA], au moins jusqu'à des champs de $5 \times 10^{7} \mathrm{Vm}^{-1}$.

La constante de vitesse d'extraction $k_{\text {ex }}$ se déduit de l'expression (2) par la relation:

$$
j=k_{\mathrm{ex}} x_{\mathrm{A}}\left[\mathrm{Pi}^{-}\right]_{\mathrm{i}} \text {. }
$$

Lorsque l'électrode n'est plus métallique, mais présente une permittivité $\varepsilon_{s}$, dans l'expression du potentiel image et donc dans celle de $G(E)$ la permittivité $\varepsilon_{1}$ du liquide est remplacée par $\varepsilon_{\text {app }}=$ $\varepsilon_{1}\left(\varepsilon_{\mathrm{s}}+\varepsilon_{1}\right) /\left(\varepsilon_{\mathrm{s}}-\varepsilon_{1}\right)=\varepsilon_{1} / \delta$. La permittivité du solide doit être prise à une fréquence reflétant l'échelle des temps des déplacements d'un ion dans le liquide. La constante de diffusion dans un liquide de viscosité $1 \mathrm{cp}$ étant environ $2 \times 10^{-10} \mathrm{~m}^{2} \mathrm{~s}^{-1}$, $\varepsilon_{\mathrm{s}}$ doit être pris à une fréquence d'environ $10^{7} \mathrm{~Hz}$. Cette valeur est en général peu différente de la valeur statique.

Pour un champ donné, les variations de $G(E)$ avec $\delta$ sont relativement faibles. Sur la figure 3 , nous donnons à titre d'exemple $G(E)$ pour une électrode métallique $(\delta=1)$ et pour une électrode métallique couverte d'un film épais de polymère $\left(\varepsilon_{\mathrm{s}}=5,5\right.$, $\delta=0,46)$. Les variations de $G(E)$ dues à $\delta$ ne deviennent notables que pour $E>10^{7} \mathrm{Vm}^{-1}$.

\section{Elaboration et caractéristiques des couches réali- sées.}

2.1 Alumine. - L'alumine compacte est obtenue par oxydation d'aluminium pur A9 dans une solution de tartrate d'ammonium à $\mathrm{pH}=5,5$, à la température ambiante, selon le procédé classique [5]. Nous avons mesuré l'épaisseur des couches par ellipsométrie et vérifié qu'elle était reliée à la tension $V_{\mathrm{f}}$ de formation de la couche (tension d'élextrolyse) par $d(\mathrm{en} \mathrm{nm})=1,4 V_{\mathrm{f}}$. En déposant sur ces films, par évaporation thermique sous vide $\left(10^{-6} \mathrm{Torr}\right)$, une contre-électrode en aluminium, nous avons réalisé des condensateurs $\mathrm{Al} / \mathrm{Al}_{2} \mathrm{O}_{3} / \mathrm{Al}$ afin de vérifier les caractéristiques électriques des couches. L'épaisseur de la contre-électrode est 2 à $3 \mu \mathrm{m}$ et sa surface est $7 \mathrm{~mm}^{2}$. Des mesures au pont alternatif (tension $\leqslant 0,1 \mathrm{~V}$ ) donnent $\varepsilon_{\mathrm{r}}=8$ et tg $\delta=8 \times 10^{-3}$ pratiquement indépendants de la fréquence $(f \leq$ $10^{5} \mathrm{~Hz}$ ), et tout à fait comparables aux valeurs connues pour de l'alumine compacte obtenue par oxydation anodique [6]. L'alumine préparée suivant d'autres techniques, présente généralement des pertes électriques plus faibles. Par exemple par pulvérisation radiofréquence, on obtient $\operatorname{tg} \delta \sim 10^{-3}$ à $1 \mathrm{kHz}$ [7].

Sur la figure 1 sont tracées les caractéristiques $I(V)$ de trois échantillons $\mathrm{d}^{\prime} \mathrm{Al}_{2} \mathrm{O}_{3}$ de différentes épaisseurs. Le courant correspond à la valeur station- 


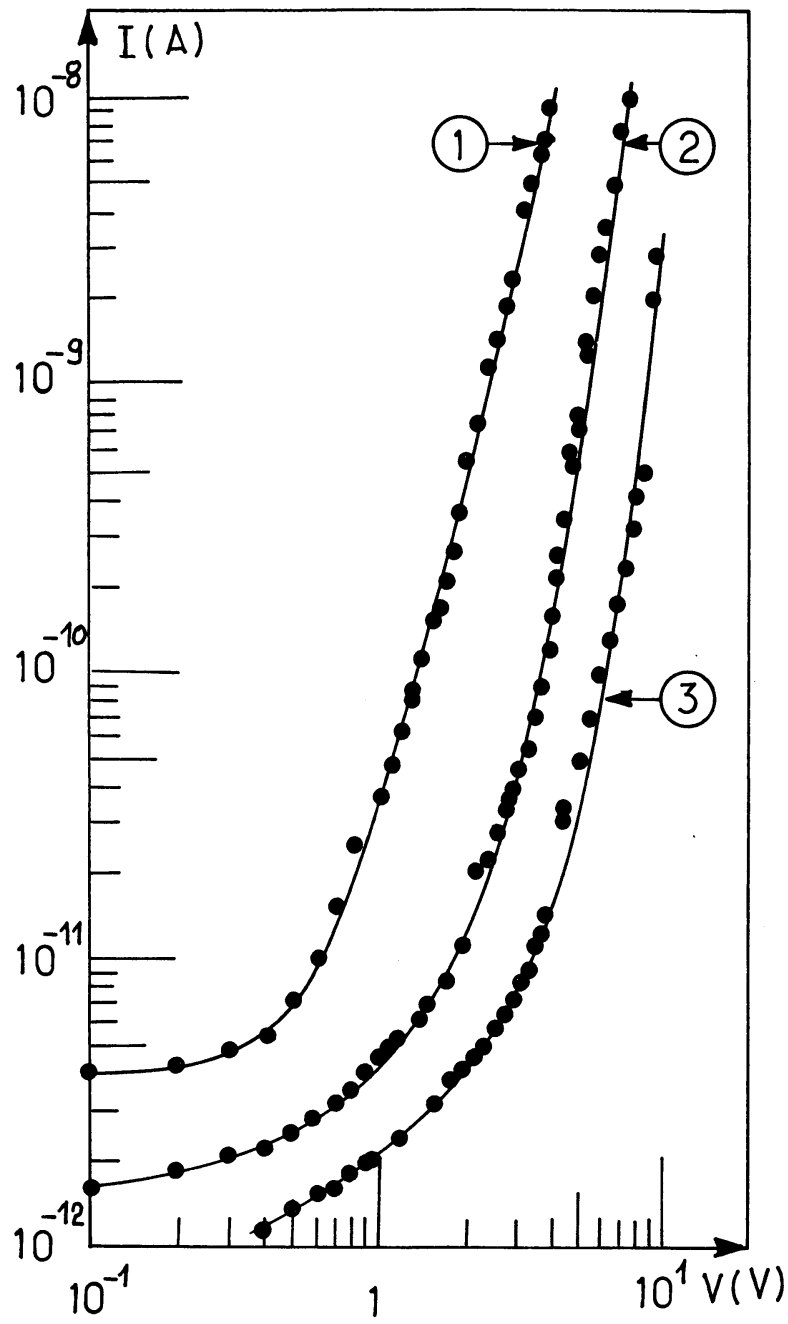

Fig. 1. - Caractéristiques $I(V)$ de structures $\mathrm{Al} / \mathrm{Al}_{2} \mathrm{O}_{3}$ pour différentes épaisseurs de $\mathrm{Al}_{2} \mathrm{O}_{3}, S=7 \mathrm{~mm}^{2}$. (1) $140 \mathrm{~nm}$; (2) $210 \mathrm{~nm}$; (3) $280 \mathrm{~nm}$.

$\left[I(V)\right.$ curves of capacitors $\mathrm{Al} / \mathrm{Al}_{2} \mathrm{O}_{3} / \mathrm{Al}$ for different thickness of $\mathrm{Al}_{2} \mathrm{O}_{3}:$ 1) $140 \mathrm{~nm}$; 2) $210 \mathrm{~nm}$; 3) $380 \mathrm{~nm}$.]

naire obtenue quelques minutes après l'application d'un échelon de tension. Pour $V>1 \mathrm{~V}$, la représentation en $j / E$ en fonction de $E^{1 / 2}$, les courbes de la figure 1 donne bien des droites mais leur pente varie entre 2,8 et $7 \times 10^{-24}$, alors que la valeur théorique relative à l'effet Schotty est $\beta_{\mathrm{S}}=\left(\mathrm{e}^{3 / 4} \pi \varepsilon\right)^{1 / 2}=$ $2 \times 10^{-24}$. Il ne nous est donc pas possible de nous prononcer sur le mode de conduction dans les couches d' $\mathrm{Al}_{2} \mathrm{O}_{3}$ que nous avons réalisées [8].

Enfin, nous avons tracé la caractéristique électrochimique d'une couche d' $\mathrm{Al}_{2} \mathrm{O}_{3}$ dans une solution électrolytique $\left(10^{-1} \mathrm{M} \mathrm{Et}_{4} \mathrm{NCIO}_{4}\right)$ dans le carbonate de propylène. On constate qu'en polarité positive, l'alumine est bloquante : la tension appliquée peut atteindre plusieurs volts sans passage notable d'un courant $(<1 \mu \mathrm{A})$. Ce très faible courant, supposé de nature électronique est attribué à la présence d'impuretés dans le film [9]. Par contre, en polarité négative, un courant important $(>50 \mu \mathrm{A})$ traverse la couche alors que la tension d'électrolyse ne dépasse que de $0,6 \mathrm{~V}$ sa valeur sur électrode d'aluminium. C'est une caractéristique de tous les films barrières de laisser passer le courant en polarité négative, même pour des champs faibles. Ce comportement est attribué $[10,11]$ à l'existence de défauts ou de fissures dans le film.

2.2 Le POLYPHENYLÈNE-OXYDE (PPOA). - Le polyphénylène-oxyde a été préparé par oxydation électrochimique de l'allylphénol en solution hydroalcoolique fortement basique, en présence d'allylamine, ce qui permet de réaliser des dépôts épais (jusqu'à $10 \mu \mathrm{m}$ ) et homogènes [12].

L'amine empêcherait la formation anodique d'oxydes métalliques qui inhibent la croissance du film de polymère [13]. L'épaisseur des dépôts réalisés varie avec la durée d'application de la tension, entre 1 et $15 \mu \mathrm{m}$. Elle a été mesurée par interférométrie. Le film est ensuite lavé à l'eau distillée puis séché sous vide à $140^{\circ} \mathrm{C}$ pendant $30 \mathrm{~min}$. Le film est alors

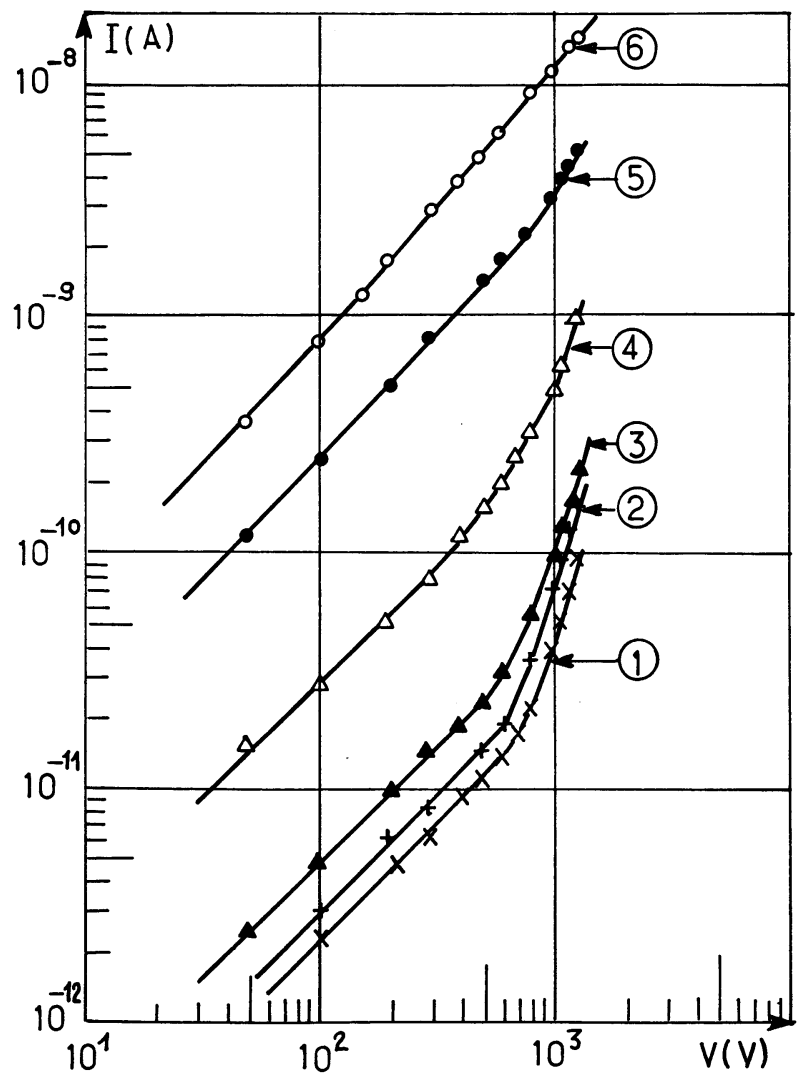

Fig. 2. - Caractéristiques $I(V)$ d'une structure, Inox/«PPOA »/inox $\left(S=2 \mathrm{~mm}^{2}, e=14,5 \mu \mathrm{m}\right)$ en fonction de la température : (1) $0{ }^{\circ} \mathrm{C}$; (2) $10{ }^{\circ} \mathrm{C}$; (3) $20^{\circ} \mathrm{C}$; (4) $50{ }^{\circ} \mathrm{C}$; (5) $80^{\circ} \mathrm{C}$; (6) $100{ }^{\circ} \mathrm{C}$.

$[I(V)$ curves of capacitors stainless steel/PPOA/stainless steel, for various temperatures : 1) $0{ }^{\circ} \mathrm{C}$; 2) $10{ }^{\circ} \mathrm{C}$; 3) $20^{\circ} \mathrm{C}$; 4) $50{ }^{\circ} \mathrm{C}$; 5) $80^{\circ} \mathrm{C}$; 6) $100{ }^{\circ} \mathrm{C} . S=2 \mathrm{~mm}^{2}$; PPOA thickness : $14.5 \mu \mathrm{m}$.] 
d'aspect brillant, il est insoluble dans de nombreux solvants comme l'acétone, les alcools, le carbonate de propylène, des hydrocarbures comme le cyclohexane, le benzène. La régularité du film et l'absence de défauts ont été vérifiées par microscopie électronique à balayage. Par réflexion spéculaire, nous avons tracé leur spectre IR. Celui-ci présente en plus des raies caractéristiques du poly(oxyde de allyl-2 phénylène-1,4) une raie caractéristique du groupement $\mathrm{NH}_{2}$. Le polymère ainsi synthétisé n'est donc pas exactement du polyphénylène-oxyde, aussi nous l'avons appelé «PPOA ».

Les propriétés électriques de ces couches ont été étudiées dans des structures métal/isolant/métal. La permittivité du PPOA est $5,5 \pm 0,5$, ses pertes diélectriques sont constantes jusqu'à environ $5 \times$ $10^{4} \mathrm{~Hz}, \operatorname{tg} \delta \sim 10^{-2}$ à $1 \mathrm{kHz}$ à température ambiante. C'est l'ordre de grandeur des pertes dans les films ainsi synthétisés [14].

Les caractéristiques $I(V)$ d'un film de PPOA de $14,5 \mu \mathrm{m}$ d'épaisseur sont données sur la figure 2 , à différentes températures. Pour $V<700 \mathrm{~V}$ environ, le courant varie linéairement avec la tension, ce qui correspond à une conductivité apparente $\sigma$ du matériau de $4 \times 10^{-13} \Omega^{-1} \mathrm{~m}^{-1}$ à $20^{\circ} \mathrm{C}$. Pour la partie des caractéristiques correspondant à la croissance rapide du courant avec la tension $(V>700 \mathrm{~V})$, le tracé des quantités $\log \left(I / T^{2}\right)$ et $\log (I / \sigma)$ en fonction de $1 / T$ semble montrer que le mécanisme de conduction du PPOA en champ intense est du type Poole-Frenkel, c'est d'ailleurs le mécanisme le plus souvent invoqué pour expliquer la conduction électrique dans des couches minces de polymères $[15,16]$.

\section{Conduction aux temps courts d'un système métal/film/liquide/métal (ou film/métal).}

Aux tous premiers instants suivant la mise sous tension continue, la tension se répartit entre le PPOA et le liquide suivant leurs capacités respectives. Compte-tenu des épaisseurs respectives des couches isolantes $\left(<300 \mathrm{~nm}\right.$ pour $\mathrm{Al}_{2} \mathrm{O}_{3}$ et $<15 \mu \mathrm{m}$ pour PPOA) et du liquide $(\sim 1 \mathrm{~mm})$, toute la tension se retrouve dans le liquide. Dans les solutions étudiées $\left(10^{-3} \mathrm{M}\right.$ AOT et $6 \times$ $10^{-4} \mathrm{M}$ TlAPi dans $\mathrm{CH}$ ), nous vérifions que le courant initial $I_{0}$ mesuré juste après l'application de la tension $V(t \sim 50 \mu \mathrm{s})$ correspond à $G V$ où $G$ est la conductance de la cellule mesurée en tension alternative de faible amplitude. Le régime transitoire qui suit l'application du créneau de tension se produit à des temps longs $(\sim \mathrm{ms})$ devant la constante de temps du circuit de mesure $(\sim \mu \mathrm{s})$. Il dépend de la nature de l'additif (AOT ou TlAPi) dans le cyclohexane, puisque celui-ci fixe le type de conduction (en volume pour l'AOT, par injection pour le TlAPi) du cyclohexane. Mais, pour une solution donnée entre électrodes isolantes, il dépend aussi de la conductibilité $\sigma$ du liquide. Le paramètre adimensionnel permettant de chiffrer cette influence est $C_{0}=\sigma d^{2} / 2 \varepsilon K V$. Il a plusieurs significations physiques. C'est le rapport du temps de transit $\left(d^{2} / K V\right)$ des ions d'une électrode à l'autre à deux fois le temps de relaxation du liquide $(\varepsilon / \sigma)$. Il est aussi le rapport du champ $\sigma d / 2 K \varepsilon$ créé par le dépôt des ions du liquide à la surface de l'isolant au champ moyen $V / d$.

Si $C_{0} \ll 1$, à l'instant correspondant au temps de transit des ions d'une électrode à l'autre, l'accumulation des ions sur les films couvrant les électrodes n'a pas encore modifié de façon notable la tension appliquée au liquide, et le régime transitoire est le même qu'entre électrodes métalliques. Si $C_{0} \gg 1$, la tension dans le liquide diminue au fur et à mesure que les ions arrivent sur les parois bloquantes, le courant décroît plus vite qu'entre électrodes métalliques.

3.1 SOlution $10^{-3} \mathrm{M}$ AOT DANS LE CYClOHEXANE. - La résistivité de la solution est $5 \times$ $10^{11} \Omega \mathrm{cm}$. La mobilité ionique des ions de l'aérosol OT dans le cyclohexane étant $6 \times 10^{-9} \mathrm{~m}^{2} \mathrm{~V}^{-1} \mathrm{~s}^{-1}$ [17], le paramètre $C_{0}$ vaut environ 2,3 pour $V=100 \mathrm{~V}$ et $d=0,5 \mathrm{~mm}$. Expérimentalement, à $100 \mathrm{~V}$, entre électrodes couvertes de PPOA, on constate effectivement que le courant décroit constamment dans le temps. Il y a variation de la tension aux bornes du liquide dès le premier transit des ions. On note donc le caractère bloquant des électrodes recouvertes de PPOA. Aux tensions plus élevées $(>1000 \mathrm{~V})$ quand $C_{0} \gg 1$, la charge accumulée sur les films ne modifie pas sensiblement le champ dans le liquide et on observe bien que le transitoire de conduction est le même qu'entre électrodes métalliques. Sa durée correspond à la mobilité connue des ions de l'AOT dans le cyclohexane. Le courant reste constant pendant au moins plusieurs temps de transit, il a la même valeur qu'entre électrodes métalliques.

3.2 Solution DE TIAPi Dans CH. - Lors de l'application d'un échelon de tension à cette solution placée entre électrodes métalliques couvertes d' $\mathrm{Al}_{2} \mathrm{O}_{3}$ ou de PPOA, le courant transitoire présente les mêmes variations dans le temps qu'entre électrodes métalliques, si la tension est telle que $C_{0}=$ $q_{0} d^{2} / \varepsilon V \ll 1$. On constate en effet une croissance linéaire du courant jusqu'à établissement après un temps $T_{\mathrm{t}}$ d'un courant stationnaire. On vérifie que $T_{\mathrm{t}}$ est bien un temps de transit $\left(\alpha d^{2} / V\right)$ la mobilité calculée est celle des ions du TlAPi dans $\mathrm{CH}$, $1,3 \times 10^{-8} \mathrm{~m}^{2} \mathrm{~V}^{-1} \mathrm{~s}^{-1}$.

La figure 3 montre la caractéristique $j(E)$ correspondant à des électrodes recouvertes de PPOA. La résistivité de la solution est relativement faible $\left(6,3 \times 10^{10} \Omega \mathrm{m}\right)$. 


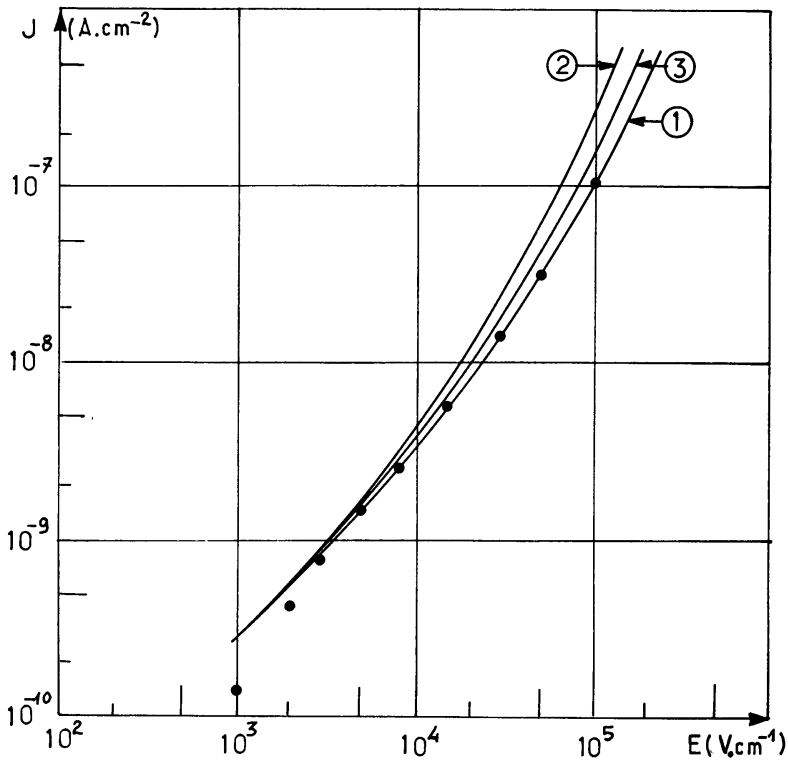

Fig. 3. - Caractéristiques $j(E)$ d'une solution $\mathrm{CH}+\mathrm{TlAPi}$ aux temps courts entre électrodes recouvertes d'un film de PPOA. $S=7 \mathrm{~cm}^{2}$, résistivité du liquide : $6,3 \times 10^{10} \Omega \mathrm{m}$; épaisseur du film : 7,3 $\mu \mathrm{m}$. Points expérimentaux ; 1 : Courbe théorique $G(E)$ relative au PPOA, $\delta=0,46 ; 2$ ) courbe théorique relative au métal; 3 : courbe théorique relative à $\mathrm{Al}_{2} \mathrm{O}_{3}, \delta=0,6$.

[Field dependence of the current density in a CH + TlAPi solution between metal electrodes coated with PPOA. $S: 7 \mathrm{~cm}^{2}$; liquid resistivity : $6.3 \times 10^{10} \Omega \mathrm{m}$; film thickness : $7.3 \mu \mathrm{m}$; Experimental points ; theoretical curves $G(E)$ relative to PPOA $(\delta=0,46)$ (curve 1), to a metal (curve 2), and to $\mathrm{Al}_{2} \mathrm{O}_{3}(\delta=0,6)$ (curve 3).]

On constate, que les points expérimentaux vérifient bien la loi théorique $G(E)$ calculée avec une permittivité apparente $\varepsilon_{\mathrm{app}}=\varepsilon_{1} / \delta$ correspondant à l'interface PPOA/CH $\left(\varepsilon_{1}=2, \varepsilon_{\mathrm{s}}=5,5, \delta=0,46\right)$ pour $E \geq 4 \times 10^{3} \mathrm{~V} \mathrm{~cm}^{-1}$ ce qui correspond à $C_{0} \leq 0,12$. Aux champs inférieurs, l'effet bloquant des électrodes se manifeste dès le premier temps de transit des ions : le courant décroît lentement dans le temps pour $t>T_{\mathrm{t}}$ et nous ne pouvons plus donner de valeur stationnaire du courant. Nous avons reporté sur la figure 3 la valeur mesurée à $3 T_{\mathrm{t}}$. On comprend donc qu'elle soit inférieure à la valeur théorique aux champs faibles.

Nous retrouvons des résultats analogues avec des électrodes en aluminium recouvertes de films d'alumine ( $>100 \mathrm{~nm}$ ). Mais dans ce cas, les points expérimentaux se situent entre les courbes relatives à la loi théorique $G(E)$ calculée pour le métal $(\delta=1)$ et pour l' $^{\prime} \mathrm{Al}_{2} \mathrm{O}_{3}(\delta=0,6)$.

Si l'on s'intéresse à la valeur de la densité de courant, pour un champ et une conductivité de la solution donnés, on constate qu'elle dépend peu de la nature du film. Le rapport $j / q_{0} G(E) K$ est compris entre 2,5 et 3 que les électrodes soient nues ou recouvertes d'alumine.
Dans le cas des électrodes recouvertes de PPOA (Fig. 3), ce rapport vaut environ 3,5. Ceci signifie que l'expression (2) de $j$ établie pour des électrodes métalliques s'applique aussi aux électrodes recouvertes de films. On déduit aussi la valeur du potentiel $\varnothing_{\mathrm{e}}$ de charge d'espace dans la couche diffuse d'ions près de l'électrode. $\varnothing_{\mathrm{e}}$ vaut de l'ordre de $1,03 \mathrm{U}$ pour les interfaces

$$
\text { métal } / \mathrm{CH}+\mathrm{TlAPi} \text { et } \mathrm{Al}_{2} \mathrm{O}_{3} / \mathrm{CH}+\mathrm{TlAPi} \text {, }
$$

il faut environ 1,25 $\mathrm{U}$ pour PPOA/CH + TlAPi.

Même si l'accord avec la théorie n'est pas parfait pour les couches $\mathrm{d}^{\prime} \mathrm{Al}_{2} \mathrm{O}_{3}$ on peut conclure après cette étude que l'injection observée aux temps courts quand les électrodes sont recouvertes de films d' $^{\prime} \mathrm{Al}_{2} \mathrm{O}_{3}$ ou PPOA, est le même phénomène que celui étudié entre électrodes métalliques. Nous reviendrons sur ce point dans la discussion.

\section{Conduction aux temps longs.}

Nous avons étudié les variations du courant lors d'une application prolongée d'une tension continue à des solutions de TlAPi dans le cyclohexane, entre des électrodes métalliques nues ou recouvertes d'un film.

Quand les électrodes sont métalliques ou quand l'anode est recouverte d'un film d' $\mathrm{Al}_{2} \mathrm{O}_{3}$ le courant garde la valeur stationnaire atteinte après le premier transit des ions injectés, pendant plusieurs heures d'application de la tension. Ceci est dû au courant très faible $\left(<10^{-6} \mathrm{~A}\right)$ dans la cellule qui ne peut causer de variations notables de la concentration en TlAPi pendant ce laps de temps. Il est surprenant de constater que le film d' $\mathrm{Al}_{2} \mathrm{O}_{3}$ que l'on s'attend à trouver bloquant à l'anode (comportement électrochimique) se comporte comme une électrode ouverte dans notre système. La raison est certainement le niveau de conduction très faible de nos solutions.

Dans le cas d'un film d' $\mathrm{Al}_{2} \mathrm{O}_{3}$ sur la cathode ou de films de PPOA sur l'anode ou sur la cathode, les observations sont similaires. Mais compte tenu des épaisseurs très différentes de ces films ( $>100 \mathrm{~mm}$ pour l' $\mathrm{Al}_{2} \mathrm{O}_{3}$ et $\sim 10 \mu \mathrm{m}$ pour le PPOA) et de leurs caractéristiques $I(V)$ mesurées entre deux électrodes métalliques, si les électrodes sont recouvertes $\mathrm{d}^{\prime} \mathrm{Al}_{2} \mathrm{O}_{3}$, la tension appliquée au liquide va rester aux temps longs peu différente de la tension totale appliquée à la cellule (erreur $\ll 10^{-3}$ ). Par contre si les électrodes sont recouvertes de PPOA, la chute de tension dans le film d'épaisseur plus importante ne pourra pas être négligée a priori. Nous séparons donc les résultats obtenus avec ces deux films.

4.1 Film D'Alumine. - Nous mesurons le courant dans une cellule contenant une solution de TlAPi dans le cyclohexane entre une électrode en aluminium et une électrode en aluminium recouverte de $140 \mathrm{~nm}$ d'alumine. Comme indiqué précédemment, 
quand l'alumine est polarisée positivement, le courant garde la valeur obtenue à $t=T_{\mathrm{t}}$ pendant plusieurs heures. Par contre, quand l'alumine est polarisée négativement, on observe des variations importantes du courant (Fig. 4). Après un temps $t_{1}$ d'application de la tension, le courant crô̂t en $A t^{n}$ avec $n \sim 0,6$ quelle que soit la tension appliquée. Le temps $t_{1}$ qui est d'autant plus court que la tension appliquée et donc le courant sont élevés, peut s'interpréter comme le temps de charge du condensateur de capacité $C$ constitué par l'alumine. En effet, si l'on calcule la tension aux bornes de $\mathrm{l}^{\prime} \mathrm{Al}_{2} \mathrm{O}_{3}$ due aux ions du liquide qui se sont accumulés pendant ce temps $t_{1}$ à la surface de $\mathrm{Al}_{2} \mathrm{O}_{3}$, on obtient $\sim 0,25 \mathrm{~V}$ indépendamment de la tension totale appliquée alors que $t_{1}$ passe de $600 \mathrm{~s}$ à $0,4 \mathrm{~s}$. Le champ dans le film n'est alors que $2 \times 10^{6} \mathrm{~V} \mathrm{~m}^{-1}$, ce qui paraît faible pour modifier la conduction dans $\mathrm{Al}_{2} \mathrm{O}_{3}$. Toutefois, c'est l'ordre de grandeur de la tension pour laquelle le courant se met à croître très rapidement dans les structures $\mathrm{Al} / \mathrm{Al}_{2} \mathrm{O}_{3} / \mathrm{Al}$ (Fig. 1).

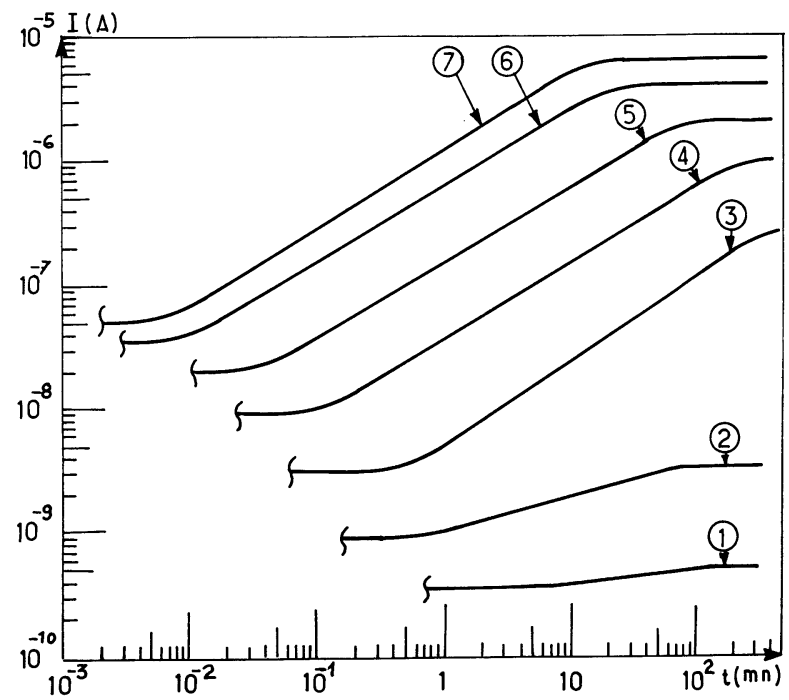

Fig. 4. - Conduction aux temps longs dans le système cathode $\mathrm{Al} / \mathrm{Al}_{2} \mathrm{O}_{3} / \mathrm{CH}+\mathrm{TlAPi} /$ anode $\mathrm{Al}$. Epaisseur de $\mathrm{Al}_{2} \mathrm{O}_{3}: 140 \mathrm{~nm}$; épaisseur de liquide : $0,94 \mathrm{~mm}$. Surface des électrodes : $7 \mathrm{~cm}^{2}$. (1) $100 \mathrm{~V}$; (2) $300 \mathrm{~V}$; (3) $1 \mathrm{kV}$; (4) $2 \mathrm{kV}$; (5) $3 \mathrm{kV}$; (6) $4 \mathrm{kV}$; (7) $5 \mathrm{kV}$.

[Variations with time of the current in a structure cathode $\mathrm{Al} / \mathrm{Al}_{2} \mathrm{O}_{3} / \mathrm{CH}+\mathrm{TlAPi} /$ anode $\mathrm{Al}$, for different voltages : 1) $100 \mathrm{~V}$; 2) $300 \mathrm{~V}$; 3) $1 \mathrm{kV}$; 4) $2 \mathrm{kV}$; 5) $3 \mathrm{kV}$; 6) $4 \mathrm{kV}$; 7) $5 \mathrm{kV}$. Thickness of $\mathrm{Al}_{2} \mathrm{O}_{3}: 140 \mathrm{~nm}$; liquid thickness : $0,94 \mathrm{~mm} ; S=7 \mathrm{~cm}^{2}$.]

Pour une tension donnée, le courant atteint une valeur stationnaire qui est imposée par le liquide. Cette valeur est en effet proche de la limitation par charge d'espace dans le liquide calculée en tenant compte des mouvements turbulents du liquide causés par la très forte injection. L'expression théorique de ce courant, représentée courbe 4 , figure 5 , est

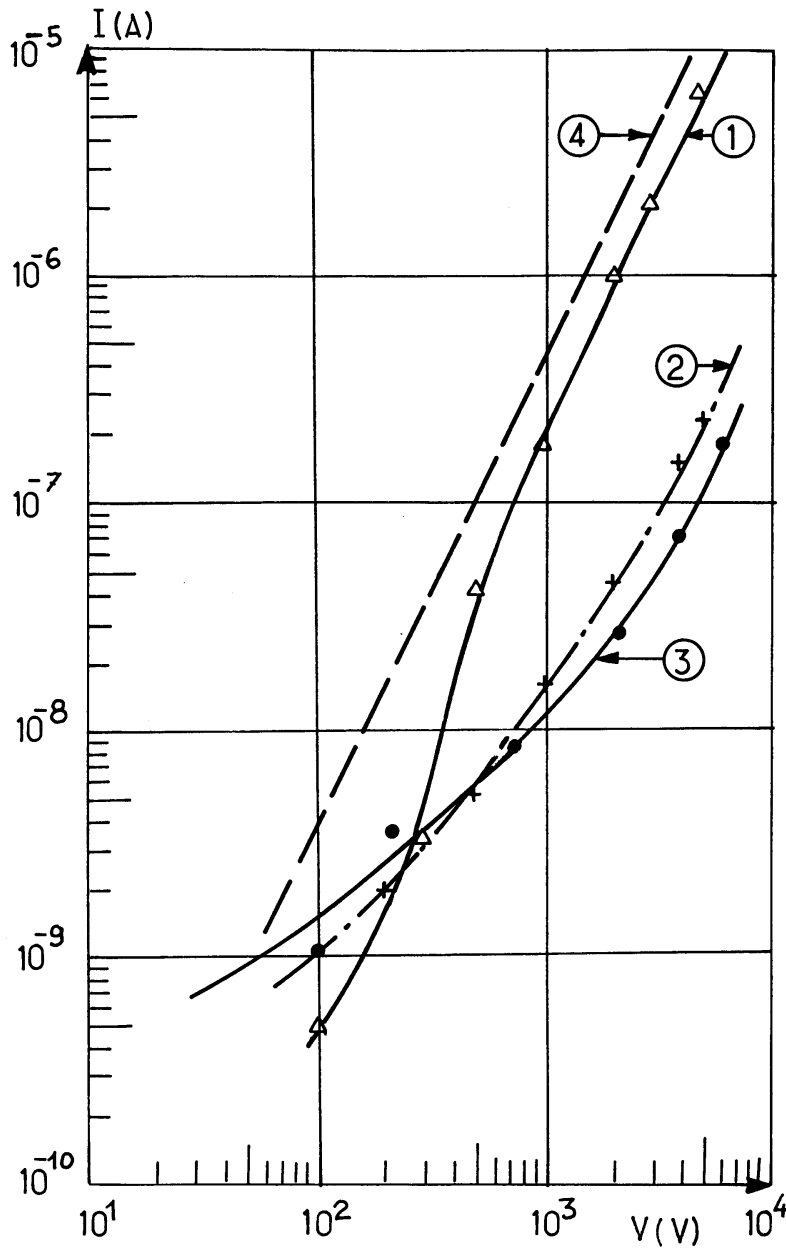

Fig. 5. - Variation avec la tension du courant stationnaire établi aux temps longs dans une structure anode $\mathrm{Al} / \mathrm{Al}_{2} \mathrm{O}_{3} / \mathrm{CH}+\mathrm{TlAPi} /$ cathode $\mathrm{Al}$. $S=7 \mathrm{~cm}^{2}$; épaisseur d' $\mathrm{Al}_{2} \mathrm{O}_{3}: 140 \mathrm{~nm}$; épaisseur de liquide : 0,94 mm. Courbe 1: $\mathrm{Al}_{2} \mathrm{O}_{3}$ à la cathode ; Courbe 2: $\mathrm{Al}_{2} \mathrm{O}_{3}$ à l'anode ; Courbe 3: électrodes métalliques nues; Courbe 4 : courbe théorique du courant limité par charge d'espace.

[Voltage dependence of the stationary current established after a long application of a voltage to a structure $\mathrm{Al} / \mathrm{Al}_{2} \mathrm{O}_{3} / \mathrm{CH}+\mathrm{TlAPi} / \mathrm{Al} ; S=7 \mathrm{~cm}^{2} ; \mathrm{Al}_{2} \mathrm{O}_{3}$ thickness : $140 \mathrm{~nm}$; liquid thickness : $0.94 \mathrm{~mm}$. Curve 1 : $\mathrm{Al}_{2} \mathrm{O}_{3}$ at the cathode; Curve 2: $\mathrm{Al}_{2} \mathrm{O}_{3}$ at the anode ; Curve 3 : metal electrodes ; Curve 4 : theoretical curve for space charge limited current with liquid convection.]

$j=\left[K K_{\mathrm{h}} / 3\right]^{1 / 2} \varepsilon V^{2} / d^{3}$, où $K_{\mathrm{h}}$ est la mobilité hydrodynamique $\left(K_{\mathrm{h}}=(\varepsilon / \rho)^{1 / 2}=1,5 \times 10^{-7} \mathrm{~m}^{2} \mathrm{~V}^{-1} \mathrm{~s}^{-1}\right)$ et $K$ est la mobilité ionique des ions du TlAPi dans le cyclohexane.

L'augmentation du courant après le temps $t_{1}$ ne peut s'expliquer que par une injection supplémentaire d'ions dans $\mathrm{CH}$ par la couche d'alumine placée à la cathode. Elle n'est observée ni entre électrodes métalliques ni quand l'anode est recouverte d' $\mathrm{Al}_{2} \mathrm{O}_{3}$. Elle vient se rajouter à l'injection (due à la présence de TlAPi dans le cyclohexane) qui se produit dès 
l'application de la tension. Nous en concluons qu'à l'interface alumine/ $\mathrm{CH}$, quand le champ dans $\mathrm{Al}_{2} \mathrm{O}_{3}$ est suffisant, il y a création d'ions par échange d'électrons entre $\mathrm{Al}_{2} \mathrm{O}_{3}$ et la solution. Ces ions viennent augmenter la densité de charge dans la zone de force-image et donc la densité du courant d'injection. Nous n'avons pas pu prouver cette hypothèse en mesurant le champ électrique dans le liquide par effet Kerr. En effet, aux tensions nécessaires pour détecter des variations de champ de 20 à $30 \%$, le claquage de la couche intervenait avant le déclenchement de l'injection. Par contre, nous avons observé un phénomène tout à fait similaire dans du carbonate de propylène entre électrodes recouvertes d'alumine. La résistivité de ce liquide polaire étant nettement plus faible $(\sim 5 \times$ $10^{10} \Omega \mathrm{cm}$ ), la croissance rapide du courant se produit quelques secondes seulement après la mise sous tension. La constante de Kerr élevée de ce liquide permet de mesurer de faibles variations de champ. Nous avons constaté que l'injection se produisait uniquement à la cathode, comme nous le supposons dans le cyclohexane.

La nature des anions injectés, le mécanisme de leur création n'ont pas été déterminés. Nous avons

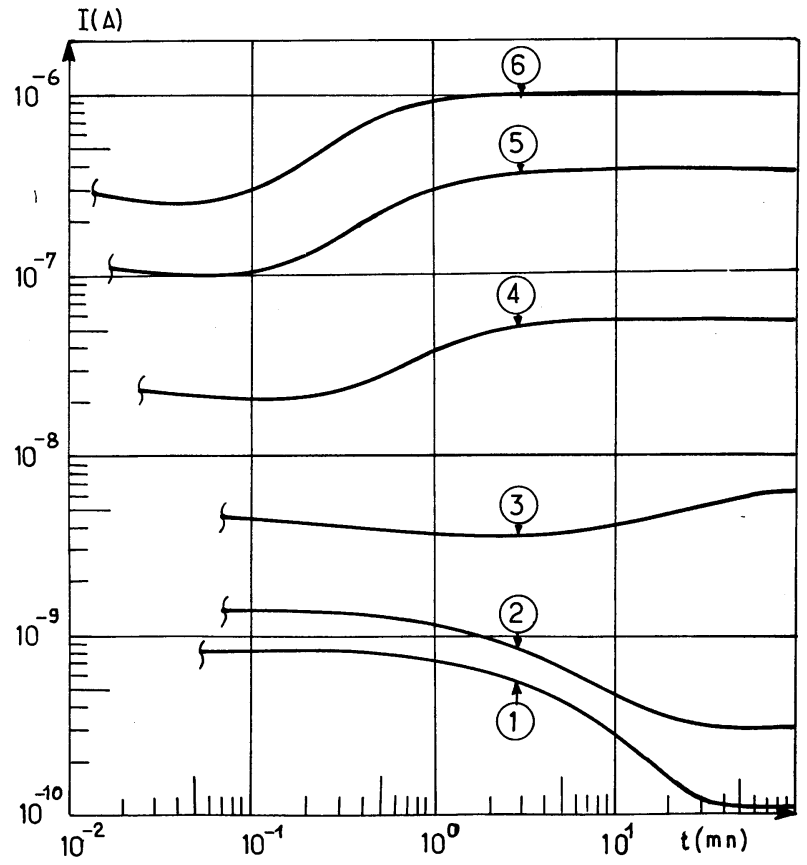

Fig. 6. - Conduction aux temps longs dans le système (cathode inox)/PPOA/CH + T1APi/anode inox. Variations dans le temps du courant pour differentes tensions : 1) $100 \mathrm{~V}$; 2) $200 \mathrm{~V}$; 3) $500 \mathrm{~V}$; 4) $1 \mathrm{kV}$; 5) $2 \mathrm{kV}$; 6) $3 \mathrm{kV}$; épaisseur du film $=8,7 \mu \mathrm{m}$; épaisseur de liquide $=0,47 \mathrm{~mm} ; S=7 \mathrm{~cm}^{2}$.

[Variations with time of the current in a structure cathode stainless steel/PPOA/CH + TlAPi/anode stainless steel, for different voltages : 1) $100 \mathrm{~V}$; 2) $200 \mathrm{~V}$; 3) $500 \mathrm{~V}$; 4) $1 \mathrm{kV}$; 5) $2 \mathrm{kV}$; 6) $3 \mathrm{kV}$. Film thickness : $8.7 \mu \mathrm{m}$; liquid thickness : $0.47 \mathrm{~mm} ; S=7 \mathrm{~cm}^{2}$.] simplement vérifié qu'après mise sous tension prolongée de la cellule, il n'y avait pas de modification décelable ni de l'état de surface de l'électrode et de ses caractéristiques diélectriques, $(\varepsilon, \operatorname{tg} \delta)$, ni de la résistivité du liquide.

4.2 FILM DE PPOA. - Sur la figure 6 sont tracées pour différentes tensions les variations dans le temps du courant stationnaire dans une solution $\mathrm{CH}+$ TlAPi quand l'anode métallique est nue et la cathode recouverte d'un film de PPOA. Contrairement au cas de l' $^{\prime} \mathrm{Al}_{2} \mathrm{O}_{3}$, la caractéristique $I(V)$ ne dépend pas de la polarité du film.

Quand la tension est inférieure à $500 \mathrm{~V}$ environ (Fig. 7, courbe 1), après le régime transitoire décrit

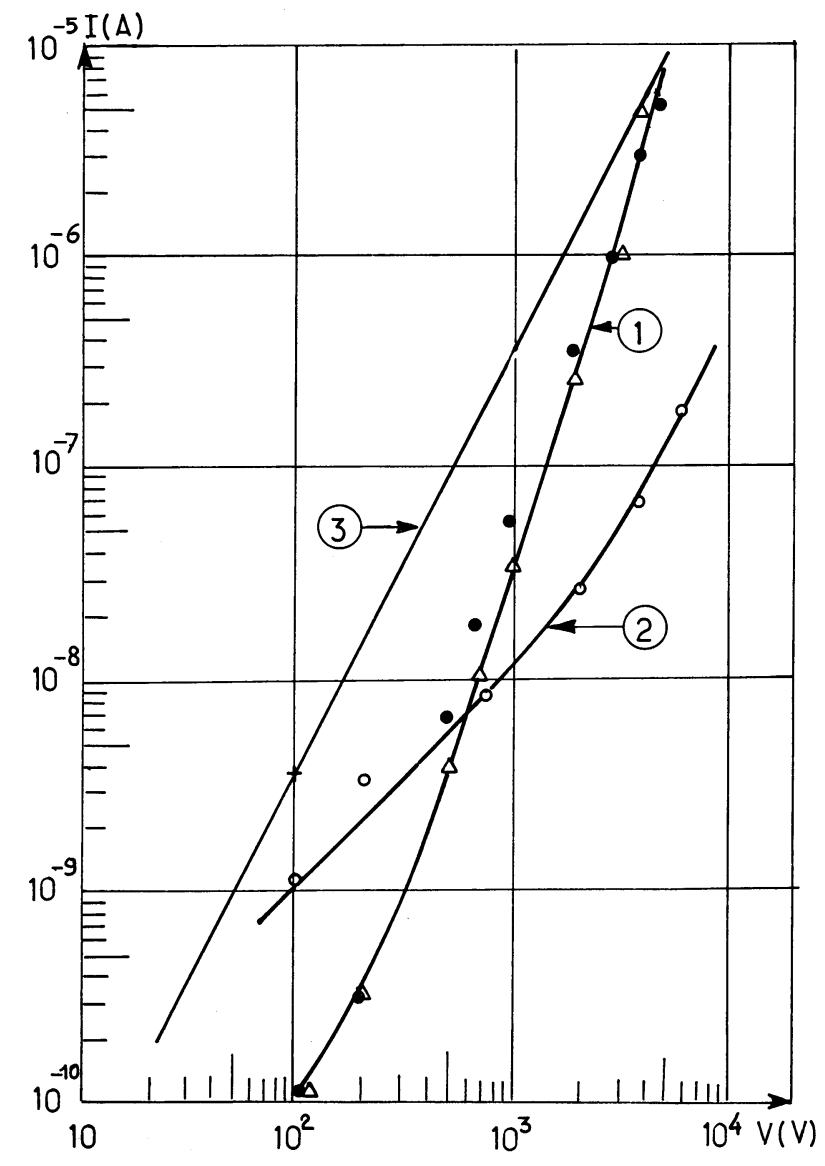

Fig. 7. - Variations avec la tension du courant stationnaire établi aux temps longs : Courbe 1 : film de PPOA recouvrant l'anode $(0)$ ou la cathode $(\Delta)$ en acier inoxydable : épaisseur du film : $8,7 \mu \mathrm{m}$. Courbe 2 : électrodes métalliques nues; $S=7 \mathrm{~cm}^{2}$; solution $\mathrm{CH}+$ TlAPi. Courbe 3: courbe théorique du courant limité par la charge d'espace.

[Voltage dependence of the stationary current established after a long application of a voltage to a structure metal/PPOA/CH + TlAPi/metal. Curve $1:$ film of PPOA at the anode $(0)$ or at the cathode $(\triangle)$ film thickness : $8.7 \mu \mathrm{m}$. Curve 2: metal (stainless steel) electrodes ; $S=7 \mathrm{~cm}^{2}$. Curve 3 : theoretical curve for space charge limited current with liquid convection.] 
dans le chapitre précédent, le courant décroît jusqu'à une valeur nettement inférieure à la valeur obtenue entre électrodes métalliques (Fig. 7, courbe 2). On vérifie aisément que l'accumulation d'ions à la surface du PPOA cause la diminution du champ électrique dans le liquide et celle du courant. Par exemple, pour une tension totale de $100 \mathrm{~V}$, un courant de $10^{-10} \mathrm{~A}$ dans le liquide dû à l'injection analysée pendant le régime transitoire suppose une chute de tension dans le PPOA d'environ $90 \mathrm{~V}$. Un calcul approché de cette chute de tension dans le PPOA due au blocage des ions sur le film donne le bon ordre de grandeur.

Quand la tension totale appliquée dépasse environ $500 \mathrm{~V}$ (Fig. 6), avec une épaisseur de liquide de $0,5 \mathrm{~mm}$ environ et un film de PPOA de $8,7 \mu \mathrm{m}$, on constate que le courant croît lentement dans le temps et prend une valeur stationnaire nettement supérieure à celle mesurée entre électrodes métalliques (Fig. 7).

A l'instant $t_{1}$ où le courant se met à croître dans le temps, on constate que la tension appliquée se retrouve essentiellement aux bornes du liquide. Par exemple, à $1000 \mathrm{~V}, t_{1} \sim 15 \mathrm{~s}$, la tension aux bornes du PPOA est environ $95 \mathrm{~V}$ dans les conditions d'essais des figures 6 et 7 . C'est une valeur maximum car le calcul suppose que le PPOA bloque les ions injectés par le métal. Cette chute de tension dans le PPOA n'est pas négligeable ; on explique ainsi que pour $t<t_{1}$ le courant diminue légèrement (Fig. 6). A cette tension correspond un champ dans le PPOA d'environ $1,1 \times 10^{7} \mathrm{~V} / \mathrm{m}$. Pour cette valeur, la caractéristique du PPOA dans une structure métal/ $\mathrm{PPOA} /$ métal reste ohmique (Fig. 2), mais on ne peut rien conclure sur le comportement de l'interface PPOA/liquide.

En tension élevée $(\sim 5 \mathrm{kV})$, la valeur stationnaire du courant est peu différente du courant limité par charge d'espace avec transport convectif de la charge. Ce courant a été évalué comme pour les expériences relatives à l'alumine, mais en supposant que toute la tension est appliquée au liquide, compte tenu de l'épaisseur d' $\mathrm{Al}_{2} \mathrm{O}_{3}$. Le claquage de la cellule ne nous a pas permis de tracer la courbe à des tensions supérieures à $5 \mathrm{kV}$.

La nature de l'additif n'intervient pas sur le comportement aux temps longs des électrodes recouvertes de PPOA. Nous obtenons les mêmes courbes $I(t)$ et $I(V)$ quand la solution de TlAPi dans $\mathrm{CH}$ est remplacée par une solution $10^{-3}$ AOT dans $\mathrm{CH}$. Toutefois, la solution d'AOT étant plus conductrice, la remontée du courant se produit à des temps plus courts. Par contre, les caractéristiques $I(V)$ du cyclohexane pur de résistivité beaucoup plus élevée $\left(>10^{14} \Omega \mathrm{cm}\right.$ ) sont identiques sur métal et sur film $\mathrm{d}^{\prime} \mathrm{Al}_{2} \mathrm{O}_{3}$ ou PPOA, aucune remontée de courant n'est observée lors d'application prolongée ( $\sim 2$ heures).
Dans tous les cas, nous n'avons détecté, dans la limite de nos moyens d'analyse, aucune modification du dépôt ou du liquide lors d'une application prolongée de la tension.

\section{Discussion.}

Nos résultats expérimentaux montrent qu'un revêtement de PPOA ou d'alumine sur une électrode métallique ne diminue pas l'injection unipolaire observée dans des solutions de TlAPi dans le cyclohexane. L'étude des phénomènes dans le temps suivant l'application d'une tension continue nous fait distinguer les temps de l'ordre de grandeur du temps de transit des ions entre les électrodes $(\sim \mathrm{ms})$ et les temps beaucoup plus longs ( $\sim$ heure).

\section{Aux temps courts.}

Quand la tension totale reste appliquée au liquide $\left(C_{0} \ll 1\right)$, nous vérifions que la densité de courant mesurée après un ou deux temps de transit varie comme la fonction $G(E)$ calculée avec $\varepsilon_{\text {app }}$ tenant compte des permittivités du film et du liquide. De plus, la densité de courant d'injection est bien proportionnelle à la densité $q_{0}$ en ions libres dans le liquide $\left(q_{0}=\sigma / 2 \mathrm{~K}\right)$, et sa valeur est celle mesurée entre électrodes métalliques nues. Nous en tirons plusieurs conséquences sur le mécanisme d'injection responsable du courant. Tout d'abord, la création d'ions qui ne dépend pas de la nature de l'électrode et dépend de la conductivité du liquide $\left(j \alpha q_{0}\right)$ a lieu dans le liquide. Entre électrodes métalliques, nous avions proposé le schéma suivant de la création d'ions (3): dissociation renforcée de l'électrolyte TIAPi dans la région de force-image, décharge d'un des ions sur l'électrode et donc libération d'un ion à l'interface métal liquide. Nos résultats entre électrodes recouvertes de films confirment cette interprétation.

D'autre part, entre électrodes métalliques nous avons montré [3] que l'étape d'extraction des ions hors de la zone de force-image et leur «injection » dans le liquide a une cinétique lente devant l'étape de créations d'ions. Ainsi pour [TlAPi] $=7 \times$ $10^{-4} \mathrm{M}$ et $x_{\mathrm{A}}=0,3 \mathrm{~nm}$, un calcul approché donne $k_{\text {ex }}\left[\mathrm{Pi}^{-}\right]_{\mathrm{i}} \sim 3 \times 10^{-3} \mathrm{M} \mathrm{s}^{-1}$ à $E=10^{7} \mathrm{~V} \mathrm{~m}^{-1}$ et $K_{\mathrm{D}}^{*}[\mathrm{TlAPi}] \sim 10^{8} \mathrm{M} \mathrm{s}^{-1} \cdot k_{\mathrm{ex}}$ et $k_{\mathrm{D}}^{*}$ sont les constantes de vitesse d'extraction des ions et de dissociation du TlAPi à l'interface métal/liquide. $k_{\mathrm{D}}^{*}$ est calculée suivant $k_{\mathrm{D}}^{*}=k_{\mathrm{D}}(0) \exp \left(2 x_{\mathrm{i}} / x_{\mathrm{A}}\right) ; x_{\mathrm{i}}$ est l'étendue de la zone de force-image : $x_{\mathrm{i}}=e^{2} / 16 \pi \varepsilon_{\mathrm{app}} U$. Le même calcul donne pour l'alumine

$$
\begin{aligned}
k_{\mathrm{D}}^{*}[\mathrm{TlAPi}] \sim 0,45 \mathrm{M} \mathrm{s}^{-1} \gg & \gg K_{\mathrm{ex}}\left[\mathrm{Pi}^{-}\right]_{\mathrm{i}}=3 \times 10^{-\dot{3}} \mathrm{M} \mathrm{s}^{-1} .
\end{aligned}
$$

Par contre pour le PPOA on obtient $k_{\mathrm{D}}^{*}$ [TlAPi] $10^{-4} \mathrm{M} \mathrm{s}^{-1}$ du même ordre de grandeur que 
$k_{\mathrm{ex}}\left[\mathrm{Pi}^{-}\right]_{\mathrm{i}}$. Contrairement à l'expérience, $j$ ne devrait pas suivre la loi $G(E)$. Nous attribuons ce désaccord à l'évolution certainement trop approximative de certains paramètres inconnus comme $x_{\mathrm{A}}$ et les concentrations à l'interface. Nous envisageons des essais avec des films de permittivité relative proche de 2 afin de vérifier que le courant d'injection ne suit plus la fonction $G(E)$. Une première constatation est qu'une injection de ce type n'a jamais été retrouvée lors d'études de films de polypropylène par exemple [19].

\section{Aux temps longs.}

Les résultats diffèrent selon la nature du film, sa polarité, son épaisseur. Toutefois, à l'exception de la couche d'alumine en polarité positive, on observe en faible tension appliquée une diminution du courant qui est alors déterminé par les caractéristiques du film, par contre en tension élevée une forte augmentation du courant traversant le système. La valeur maximum atteinte correspond bien à la valeur attendue pour un courant d'injection limité par charge d'espace et calculé avec une mobilité hydrodynamique apparente des ions. Les films étudiés injectent donc des ions dans le liquide. L'origine et la nature des ions n'ont pas été déterminées. Nos résultats semblent bien s'interpréter par une accumulation des ions du liquide à la surface du film qui renforce le champ électrique dans le film. Le fait surprenant est que le film devienne injecteur pour des valeurs relativement faibles du champ dans le film $(\sim 1,8 \times$ $10^{6} \mathrm{~V} \mathrm{~m}^{-1}$ pour l'alumine et $1,1 \times 10^{7} \mathrm{~V} \mathrm{~m}^{-1}$ pour le PPOA). Mais à ce stade de l'étude, nous avons encore peu d'informations sur le mécanisme d'injection par le film et les ions injectés.

\section{Bibliographie}

[1] Denat, A., Gosse, B., Gosse, J. P., J. Electrostatics, 11 (1982) 179.

[2] Willig, F., Chem. Phys. Lett. 40 (1976) 331.

[3] Alu, A., Gosse, J. P., Gosse, B., Denat, A., Nemamcha, M., Revue Phys. Appl. 22 (1987) 1041.

[4] Nemamcha, N., Gosse, J. P., Denat, A., Gosse, B., I.E.E.E. Trans. Electr. Insul. accepté pour publication.

[5] Hass, G., Y. Opt. Soc. Amer. 39 (1949) 532.

[6] KAO, K. C., HWANG, W., Electrical transport in solids (Pergamon Press) 1981.

[7] Harrop, P. J., CAMPBell, D. S., Handbook of thin films Technology (M. C. Graw Hill Company) 1970.

[8] Ikonopisov, S., Elenkov, J. Electroanal. Chem. 86 (1978) 105.

[9] Goruk, W. S., Young, L., Zobel, F. G. R., Modern Aspects of Electrochem., N ${ }^{\circ}$, Ed. J. O’M. Bockris (London Plenum Press) 1966.
[10] Vermilyea, D. A., Anodic Films, Adv. in Electrochemistry and Electrochemical Eng., Vol. 3, Ed. P. Delahay (Interscience Publisters, New-York) 1963.

[11] Young, L., Trans. Faraday Soc. 55 (1959) 842.

[12] Mengoli, G., Adv. Polym. Sci. Springer-Verlag Berlin, 33 (1979) 1.

[13] Mengoli, G., Daolid, S., Musiani, M. M., J. Appl. Electrochem. 10 (1980) 459.

[14] Dubois, J. E., Tourillon, G., Pham, M. C., LACAZE, P. C., Thin Solid Films 69 (1980) 141.

[15] Castonguay, J., Theoret, A., Thin Solid Films 69 (1980) 85.

[16] BAHri, R., Singh, H. P., Thin Solid Films 69 (1980) 281.

[17] Denat, A., Gosse, B., Gosse, J. P., Revue Phys. Appl. 16 (1981) 673.

[18] Gosse, J. P., Gosse, B., Denat, A., Rev. Gen. Electr. 10 (1985) 733.

[19] SAAD, A., Thèse de Doctorat d'Etat, Grenoble, 1986. 